\title{
THE (UlTRA)SOUND OF THINGS TO COME
}

\author{
Dr Carlos Cabalag MBBS M,2 $^{1,2}$ \\ ${ }^{1}$ Editor, Journal of Mobile Technology in Medicine, ${ }^{2}$ Monash Medical Centre, Melbourne, Australia \\ Corresponding Author: carlos@journalmtm.com \\ Journal MTM 1:1:5-7, 2012 \\ doi:10.7309/jmtm.3
}

The use of mobile technology in medicine is not limited to the tablet computer or the use of the everpopular Apple ${ }^{\circledR}$ iPhone. As semi-conductors become smaller, various devices are being redeveloped and transformed into portable miniature versions of their old previously large and cumbersome counterparts. In particular, we have seen the advancement and miniaturisation of piezoelectric transducers in the recent decade, leading to the development of portable ultrasound machines that can be utilised virtually anywhere and in settings of dire need. The most tragic and recent of those events was the Haitian earthquake in 2010, where 230,000 people lost their lives with many more injured in a background of crucial infrastructural destruction that was seemingly endless to the naked eye. ${ }^{1}$ Notably, one of the devices that had shaped the humanitarian effort to save lives, was (and still is) the humble portable ultrasound. (Refer to Figure 1). Are clinicians ready for the next diagnostic tool, some branding it as the next 'pocket stethoscope'?

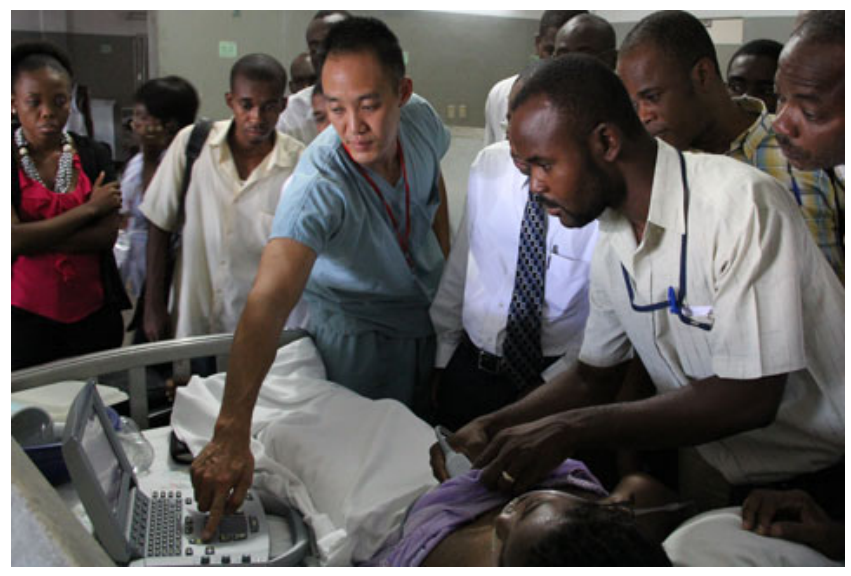

Figure 1: Trauma team using portable ultrasound in Port $\mathrm{Au}$ Prince, in the 2010 earthquake. Courtesy of internationalmedicalcorps.org.

The use of the portable ultrasound has played an instrumental role in the humanitarian effort in Haiti. Dr. Sachita Shah, an emergency physician of Rhode Island Hospital and one of the key players in the rescue effort, states the use of portable ultrasound in "mass casualty settings" as well as rapid triaging of patients for subsequent surgical or medical management. $^{2}$ Their team used the Sonosite Micromaxx $^{\mathrm{TM}}$ device to rapidly diagnose intraperitoneal and intrathoracic haemorrhage as well as various other uses from pregnancy assessment to ultrasound guided regional anaesthesia. Studies originating from the war in Iraq have also seen the use of portable ultrasound in austere medical environments specifically demonstrating the utility of FAST (Focused Assessment with Sonography in Trauma) scanning. One large study from a mobile combat support hospital scanned 400 patients within their first 6 months of operations with a hand-held portable device. Their study noted that the use of portable ultrasound improved their diagnostic accuracy as well as prevented unnecessary transfer of soldiers to tertiary medical centres for further management. ${ }^{3}$ Closer to home, the FASTER (Focused Assessment with Sonography in Trauma during Emergency Retrieval) trial, a collaboration by South and Western Australian hospitals established that it was feasible for emergency physicians to conduct inflight FAST scans using portable ultrasounds on critically ill patients en-route to definitive care, thus preventing further delays in diagnosis. ${ }^{4}$

What is the role of portable ultrasounds in the ward hospital environment? Moving to a very different clinical setting, a current cross sectional study by Liebo et al. comparing echocardiograms taken by a 'pocket mobile device' (VScan GE $\AA$ Healthcare, Milwaukee) (Figure 2) and a standard trans-thoracic echocardiogram (TTE) demonstrated highly correlative findings in most cardiac function measurements. $^{5}$ Within the constraints of a single centre study, they found that pocket mobile echocardiography (PME) yielded accurate assessments of ejection fraction and cardiac function, however there was a variance in accuracy with mitral valve abnormalities and inferior vena cava size. Interestingly, a similar comparison study using a 


\section{r. JMTM}

different pocket echocardiograph (Acuson P10, Siemens ${ }^{\circledR}$, Mountain View, CA) showed that there was a good correlation with standard TTE to quantify ejection fraction. ${ }^{6}$ Both studies demonstrate a stronger association between PME and TTE for the experienced echocardiographer.

The aforementioned studies and many more demonstrate the nascent capabilities of portable ultrasound in the clinical setting. Nevertheless, what is lacking are large multi-centre comparative studies identifying validity in comparison to the gold-standard, but also comparative costs, operator-reliability and a cost-benefit analysis with standard machines. The Vscan GE device mentioned above costs US\$7,900, which is a small margin in comparison to the standard machines that would cost in excess of US\$30,000. This, at first, may appear to be a substantial costsaving however one must consider the cost of training physicians to an appropriate skill level that would screen examined patients and reduce the need for unnecessary trans-thoracic echocardiograms. Training doctors in mobile bedside echocardiography may not only reduce the workload for dedicated trans-thoracic echocardiogram services in public hospital, but facilitate earlier diagnosis, and thereby ensure timely management decisions. There is also the issue of the willingness of junior medical officers as well as senior doctors alike to learn a new skill and accepting it as part of the routine physical examination of a patient.
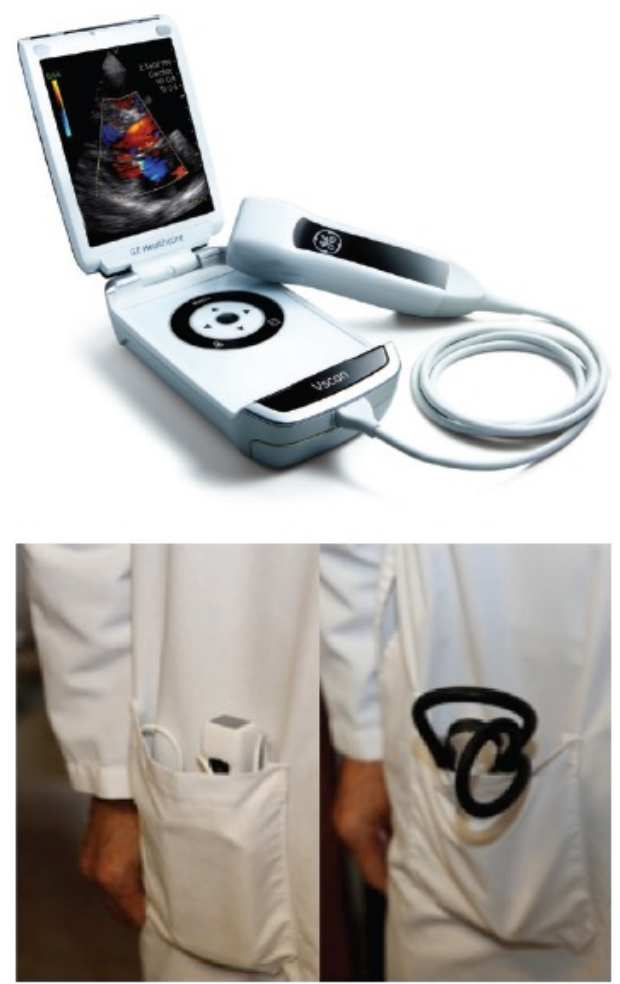

The Vscan device is manufactured by GE Healthcare, Milwaukee, Wisconsin.
Figure 2: Pocket mobile echocardiography device. Image courtesy of VScan GE® Healthcare, Milwaukee.

Perhaps a way of introducing portable ultrasound to junior doctors would be to embed its use in the learning of anatomical sciences. Ivanusic and others investigated perceptions of undergraduate students in the use of ultrasonography for the study of 'living anatomy'. Ninety percent of medical students strongly agreed that utilising trans-thoracic echocardiography to reinforce cardiac anatomy was an 'effective' teaching method as well as a sound reinforcer of the material learned. ${ }^{7}$

After discussing the economics, utilisers and politics of the use of portable ultrasound, one should also consider the technical and hardware viability especially to survive the 'elements' in severe environments. There have been case reports of portable ultrasounds deployed in extreme conditions from the Amazonian jungle to the International Space Station - the salient issues in regards to usage were the weight of the device and battery power. ${ }^{8}$ We were unable to obtain the operative limits in terms of environmental conditions however an unexpected degradation in battery life was noted in high temperature environments. Moreover, batteries being the only means of power in the absence of electricity was especially important in high-altitude environments demonstrated in Himalayan missions. ${ }^{910}$

In summary, to rationalise the use of portable ultrasound in mass casualty situations such as environmental disasters is strengthened by the fact that it aids in prompt triaging of patients as well as its role in anaesthesia. Still in contention however, is its practicability in the modern, resource-full clinical setting to aid in the bedside examination of patients. The introduction of its use in tertiary hospitals must be balanced with the cost in its initial purchase, the education of medical staff, its potential cost-savings in reducing the workload of the echocardiography department, and not to mention its propensity to optimise patient management in the ward-setting. By the 'sound' of things to come, it may be inevitable that the pocket ultrasound will be part of the clinician's armamentarium alongside the humble stethoscope.

\section{References}

1. RCA. Haiti Earthquake Appeal, 2010. http://www.redcross.org.au/haiti-earthquake-appeal2010.aspx: Red Cross Australia, 2010.

2. Shah S, Dalal A, Smith RM, Joseph G, Rogers S, Dyer GS. Impact of portable ultrasound in trauma care after the 


\section{Fi) MTM}

Haitian earthquake of 2010. Am J Emerg Med 2010;28(8):970-1.

3. Rozanski TA, Edmondson JM, Jones SB. Ultrasonography in a forward-deployed military hospital. Mil Med 2005;170(2):99-102.

4. Mazur SM, Pearce A, Alfred S, Goudie A, Sharley P. The F.A.S.T.E.R. trial. Focused assessment by sonography in trauma during emergency retrieval: a feasibility study. Injury 2008;39(5):512-8.

5. Liebo MJ, Israel RL, Lillie EO, Smith MR, Rubenson DS, Topol EJ. Is pocket mobile echocardiography the nextgeneration stethoscope? A cross-sectional comparison of rapidly acquired images with standard transthoracic echocardiography. Ann Intern Med 2011;155(1):33-8.

6. Culp BC, Mock JD, Chiles CD, Culp WC, Jr. The pocket echocardiograph: validation and feasibility. Echocardiography 2010;27(7):759-64.

7. Ivanusic J, Cowie B, Barrington M. Undergraduate student perceptions of the use of ultrasonography in the study of "living anatomy". Anat Sci Educ 2010;3(6):31822 .
8. Nelson BP, Melnick ER, Li J. Portable ultrasound for remote environments, Part I: Feasibility of field deployment. J Emerg Med 2011;40(2):190-7.

9. Fagenholz PJ, Gutman JA, Murray AF, Noble VE, Thomas SH, Harris NS. Chest ultrasonography for the diagnosis and monitoring of high-altitude pulmonary edema. Chest 2007;131(4):1013-8.

10. Fagenholz PJ, Gutman JA, Murray AF, Noble VE, Camargo CA, Jr., Harris NS. Optic nerve sheath diameter correlates with the presence and severity of acute mountain sickness: evidence for increased intracranial pressure. J Appl Physiol2009;106(4):1207-11.

\section{Disclaimer}

All product names, logos, brands and other trademarks featured or referred to within the Journal of Mobile Technology in Medicine e-publication remain the property of their respective trademark holders.

These trademark holders are not affiliated with the Journal of Mobile Technology in Medicine, and do not sponsor or endorse this journal. 